\title{
PLANNING THE PUBLIC TRANSPORT SYSTEM IN MUCAT (OMAN)
}

\author{
Juan Francisco Gonzalez Jiménez \\ Technician, Policy and Planning Area, Ineco, España \\ Eva Hitado Hernández \\ Technical Manager, Policy and Planning Area, Ineco, España \\ Carolina Sanz Pecharromán \\ Technical Manager, Policy and Planning Area, Ineco, España
}

\begin{abstract}
In Muscat, the capital of Oman with over one million inhabitants, urban public transport practically does not exist and the use of private cars is dramatically increasing. As a result, accident rates and traffic congestion, two particularly pressing concerns in the urban area of the capital, have both risen. This situation has focused the concern of public administrations and has led to the urgent need of developing a Public Transport System in the city
\end{abstract}

In order to develop this system in an integrated manner, the plan proposes to unify and organize regulation and management of public transport by creating a public transport authority, headed by the Ministry of Transport and with a very strong involvement of Muscat Municipality.

By 2015 only two urban bus lines operate in Muscat, covering a small area of the cityWadi Adei, Wadi Kabir and Ruwi. Therefore, the plan adopts a conservative approach by proposing the implementation of a comprehensive urban bus network -composed by trunk and feeder lines adapted to the low density of the city- before establishing other high capacity solutions, such as BRT or LRT.

A key element of the system is the number of 13,400 taxis and microbuses that represent the only alternative to the 317,000 cars of Muscat. The plan suggests the reorganization and further regulation of the sector to achieve the professionalization of the taxi services and the migration of existing users, part of them to the professionalised taxi services and the other part to the implemented public transport services.

\section{INTRODUCTION}

The Sultanate of Oman, the fifth largest economy of the Arabian Peninsula, has experienced an economic and social upturn since 1970's, which has entailed the development of a modern country. In recent years, the Sultanate has made substantial investments in transport infrastructure as part of the overall strategy of diversifying its 
economy, which revolves around the production and export of natural gas and oil.

Oman is home to a population of over 4 million people, of which $30 \%$-around 1.2 million people- live in the capita Muscat, in the north of the country. Muscat has then over one million inhabitants, of which around $40 \%$ are Omani national and $60 \%$ are expatriates, more than 90\% from Bangladesh, India and Pakistan, but also from other Asian countries like Indonesia, Philippines, Ethiopia or Egypt.

The population of Muscat makes an extensive use of private vehicles to get around, showing increasing levels of car dependency. According to the Royal Oman Police figures, the number of registered vehicles has increased constantly at a growth of $9.3 \%$ every year. As a result, accident rates and traffic congestion, two particularly pressing concerns in the urban area of the capital, have both risen. Due to the practically inexistence of public transport, those with no means to afford a private vehicle move around by using informal transport (taxis and microbuses) or previously arranged company buses for workers.

Within this framework, the Ministry of Transport and Communications of Oman entrusted Ineco with the drafting of a Public Transport Master Plan (PTMP) for Muscat in order to achieve a modern and efficient public transport system. The Vision of the PTMP is the development of a high quality and sustainable Public Transport system in Muscat as a real alternative to private cars, in order to reduce automobile dependency and to improve accessibility, safety, urban environment and quality of life in Muscat by achieving a 25\% public transport modal split by the year 2040 .

\begin{tabular}{|c|c|c|c|}
\hline Nationality & Inhabitants & $\% 1 \%-1 \%$ & \\
\hline Bangladeshis & 651.301 & $4 \%$ & - Bangladeshis \\
\hline Indians & 641.225 & & Indians \\
\hline Pakistanis & 216.167 & & — Pakistanis \\
\hline Indonesian & 37.264 & $12 \%$ & - Indonesian \\
\hline Philipinos & 32.482 & & — Philipinos \\
\hline Ethiopian & 28.099 & & Ethiopian \\
\hline Egyptians & 22.810 & & Egyptians \\
\hline Sri Lankas & 14.252 & & 『Sri Lankas \\
\hline Nepalese & 12.892 & & Nepalese \\
\hline Other nationalities & 65.068 & & घthe Nationalities \\
\hline Total & 1.631 .560 & & \\
\hline
\end{tabular}

Table 1 - Expatriates nationality (June 2015)

\section{STUDY METHODOLOGY}

The geographic area of study for the PTMP was the Governorate of Muscat, one of the 11 governorates into which the Sultanate is divided, which spans an area of $35,000 \mathrm{~km}^{2}$ and $200 \mathrm{~km}$ of coastline. It comprises six municipalities or districts: Muscat, Bawshar, As Seeb, Mutrah, Al Amerat and Quriyat. 
The study has followed a methodology divided into three phases. Firstly, at the early stages of the Project, a data collection and analysis of the existing situation allowed a diagnostic, carried out in order to achieve a comprehensive understanding of the functioning of the public transport in Muscat. Then, the global objectives to be achieved by the PTMP were established in coordination with the Ministry of Transport and Communication. Also, a global mobility strategy was defined regarding the transport sector and mobility, environmental and economic guidelines. Finally, Action programs were defined, with specific measures designed to meet the objectives previously defined, for each area of study. This methodology was applied to the 6 areas in which the study was organised. These areas covered all topics related to public transport.
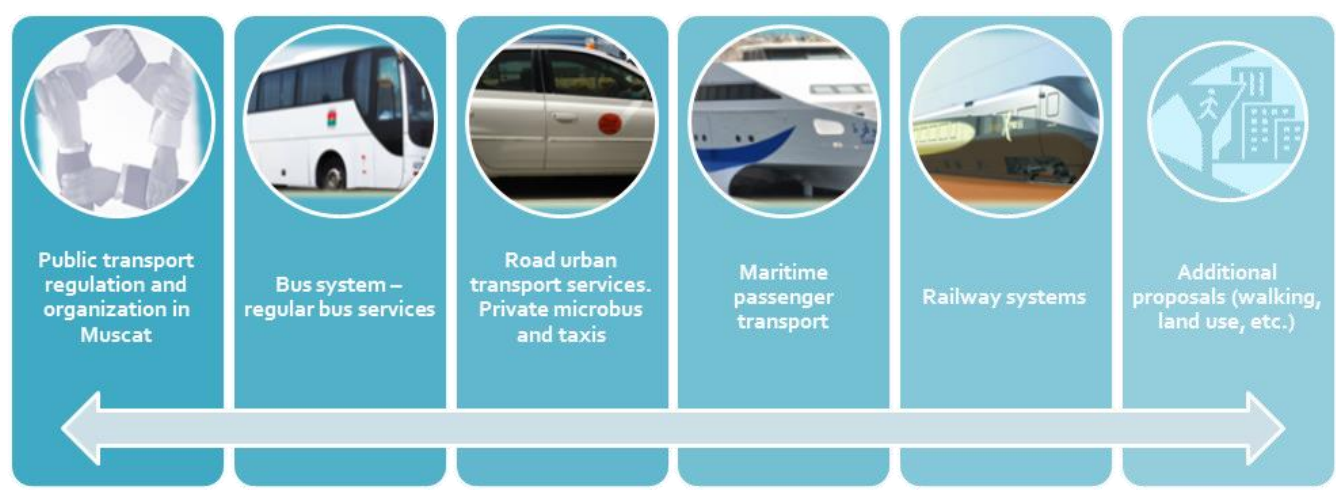

Figure 1 - Study methodology: areas and phases of study

\section{DIAGNOSTIC: A PRACTICALLY NON REGULATED AND INEXISTENT PUBLIC TRANSPORT SYSTEM}

\subsection{Weak institutional framework and organisation to develop public transport}

In Muscat there are a myriad of national and municipal entities and bodies linked to transport or with transport-related competencies. The main body is the Ministry of Transport and Communications but likewise important are the Muscat Municipality, for road-building in the capital area; along with the Royal Police of Oman, for traffic regulation and road-safety; the Oman National Transport Company, which runs the bus service; and the National Ferry Company, among others.

There is no clear organization responsible for mid and long - term plans regarding public transport or for coordinating the different institutions involved in public transport. This lack of organisation causes an overlap and duplication between organizations.

Therefore, it was detected a need of establishing an appropriate institutional framework in which the different elements of the public transport system would rely on.

\subsection{Lack of an extensive and quality public transport system in Muscat}

A lack of an extensive and quality public transport system in Muscat was detected during the diagnostic stage of the study. The existing public transport system in Muscat only covers a small area of Muscat with only two routes: Ruwi -Wadi Kabir and Ruwi - Wadi 
Adei. Both routes, as a whole, transported more than 1,2 million passengers in 2014, and ridership on its busiest route (Ruwi - Wadi Kabir ) approaches 1 million annual passengers in this period.

Taking into account that Muscat Governorate's population was in 2014 approximately 1.216.000 inhabitants, the ratio "annual bus trips/inhabitant" is about 1 . This value is extremely low compared to the recording ones in nearby cities, such as Dubai and Abu Dhabi, which have around 44 annual trips by bus per inhabitant. In Spain the most populated cities show values from 45 to 70 annual trips by bus per inhabitant. But the ratio "annual bus trips/inhabitant" is extremely low due to the practically inexistence of an extensive public transport network in Muscat, since the total number of $\mathrm{km}$ of the public transport network in Muscat, that is, the sum of the lengths of these two routes, is nearly 25 $\mathrm{km}$, which is extremely low considering the inhabitants and size of the city. However, the ratio between annual trips and route length is relatively high and shows a great potential for the extension and implementation of public transport services.

What is even more significant is that this positive ratio is achieved with very low levels of reliability and quality of services; with a great number of cancelled services (between 12 and $24 \%$ ) due to high levels of absenteeism, affecting the quality and recurrence of potential regular users; with an old fleet (more than 15 years old); with no passenger information systems, etc.

Regarding the infrastructure, bus stops do not offer sheltered and conditioned spaces for waiting, which is very important considering the extreme weather conditions of the country, and travel times are severely affected by traffic congestion. Also, the previously mentioned lack of institutional framework has resulted for example in the existence of different types of bus stops for different types of services, which creates confusion among users.

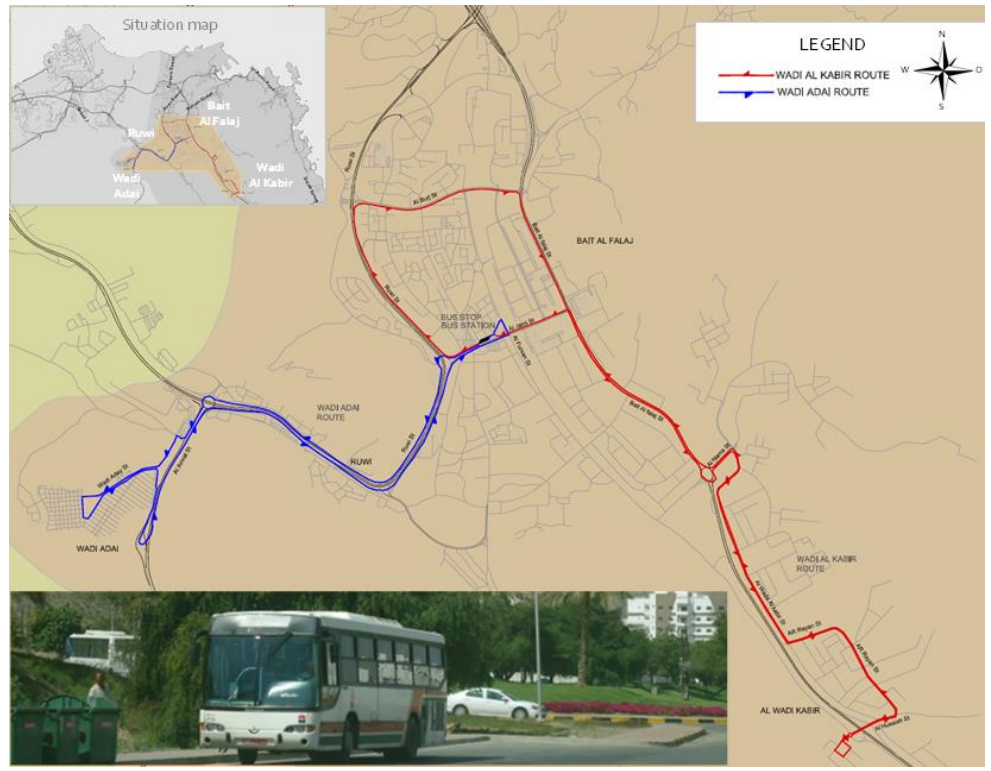

Figure 2 - Urban bus services in Muscat: uwi -Wadi Kabir and Ruwi - Wadi Adei 


\subsection{Transport needs are covered by private cars and informal transport: taxis and microbuses}

A key element of the transport system of Muscat are taxis and microbus. According to the Royal Oman Police there are a total number of 13,400 taxis and microbuses operating in Muscat, which is an extremely high number considering the population of Muscat. For example, the number of taxi licenses in Madrid is 15,700 for a population of more than 5 million and in Dubai the number is 8,702 for a population of slightly above 2 million. Therefore, it can be concluded that the total number of taxi licenses in Muscat and the number of licences per inhabitant in Muscat, compared with other international cities, is considerably high. This fact suggests several issues: the relevance of taxis in providing transport services due to the lack of public transport; the taxi sector is oversized; a lack of a professionalization in the sector, with a great number of taxi license holders not driving a taxi as a full professional activity, but rather a complementary activity, with its corresponding affections to the quality of the service.

Actually, taxis and microbuses can be considered as an informal public transport system and the only alternative to the 317,000 cars of Muscat.

Although there are many different self-organized committees of taxis for the provision of specific services - in hotels, malls or the Sultan Qaboos Port - the most common activity of taxis is roaming along the main streets of Muscat collecting and transporting passengers to different destinations on a shared basis. Users share taxi rides, even though they do not know each other and have different origins and destinations, since taxis stop to collect different people during the service. Fares or timetables are not regulated and regular user of this type of services is low income expatriates that cannot afford to own a private car. Therefore, taxis and microbuses are the only mean of transport for those who cannot afford a private vehicle due to the practically inexistence of public transport.

\section{ACTIONS TO DEVELOP A WELL-ORGANIZED PUBLIC TRANSPORT SYSTEM}

\subsection{Implementation of a robust institutional framework}

In order to develop this system in an integrated manner, it is firstly required to design an appropriate institutional framework in which the different elements of the system would rely on. The plan proposes to unify and organize regulation and management of public transport by creating a Public Transport Authority (PTA) headed by the Ministry of Transport and with a very strong involvement of Muscat Municipality. This would allow eliminating current duplication and overlapping of roles. As a consequence, by legally establishing the PTA with clear objectives, functions and responsibilities, this ambiguity and apparent overlap of functions would be clarified. This will increase the efficiency of the public transport system and will help to provide faster and more coordinated solutions. 
But also, the PTA with adequate training and knowledge of technical solutions for public transport will help to boost public transport in Muscat, by having a single empowered authority with the only responsibility of providing high quality public transport to citizens. Therefore, one single authority, the PTA, will be required to coordinate all transport modes, maximizing in this way public expenditure and achieving full integration, independently of the existence of various transport operators, which may have the responsibility for the actual operation and running of the services.

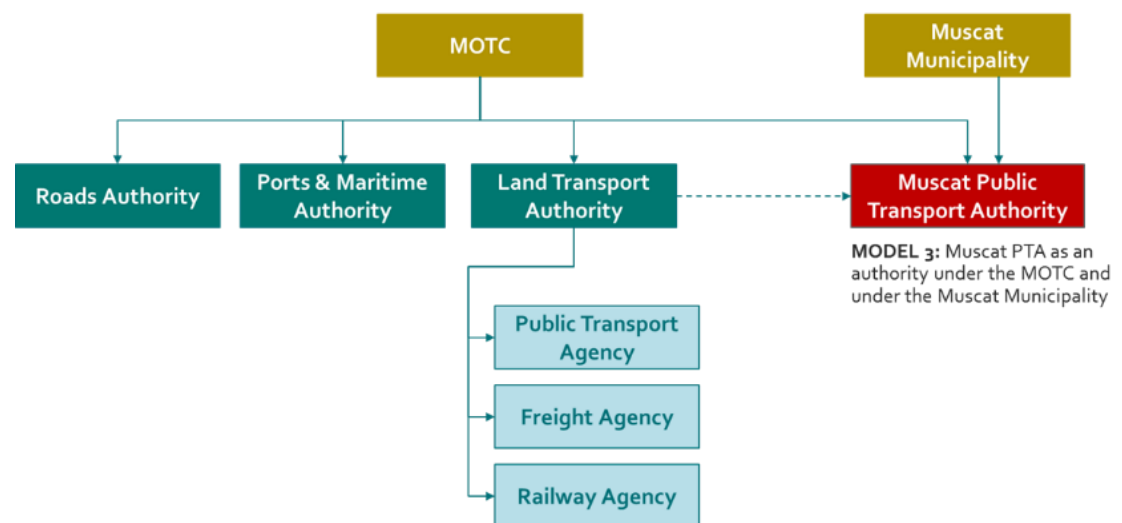

Figure 3 - Proposed recommended model for Muscat PTA

\subsection{Definition of an integrated and phased public transport network}

Due to the limited experience and current low use of public transport in Muscat, an initial system based on urban bus transport was initially recommended. This solution would allow a gradual change in the current public transport system and a better adjustment between supply and demand of public transport. Buses need to be considered as a modern way of transport, with several fuel options that allow low pollutant levels, ideal for medium populated cities, with low density of population rates as Muscat.

For these reasons, the following phases for the development of the Integrated Public Transport Network of Muscat were recommended considering that the introduction of the different systems should not be based on a temporal scale, but on demand parameters.

The recommended phased development of the network is:

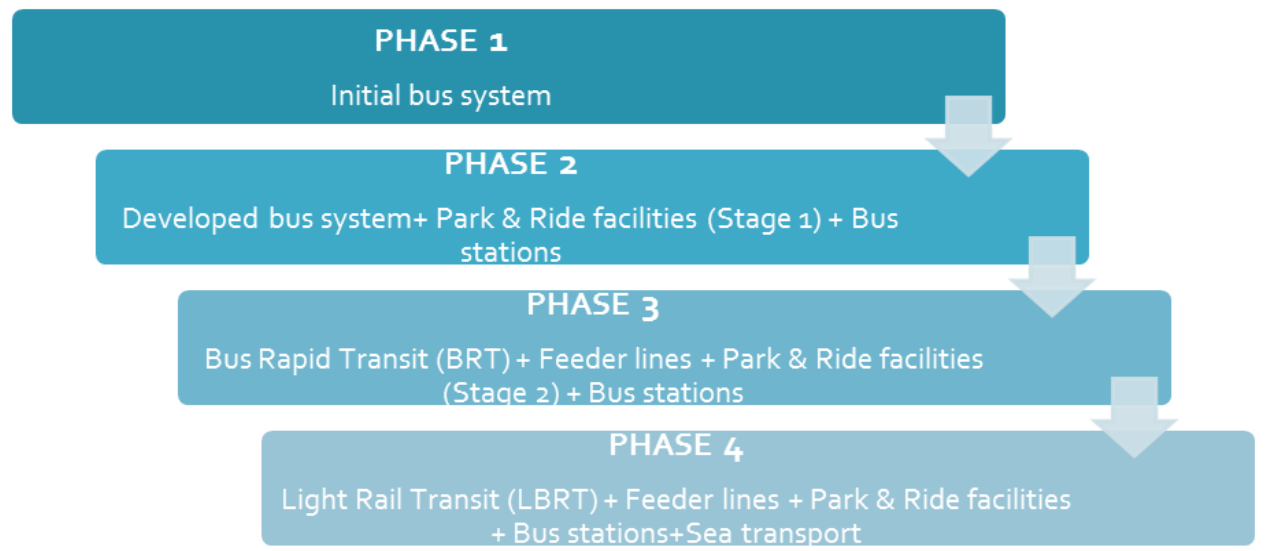

Figure 4 - Proposed network evolution 
An Integrated Public Transport Network was conceived according to the characteristics of Muscat which are the following:

- Linear city growing alongside the main street of the city, Sultan Qaboos St.

- Location of main activity centres alongside the Sultan Qaboos St.

- The identified mobility by taxis and microbuses.

- Land use pattern.

- Existing traffic characteristics of Muscat.

For these reasons, the network structure that was recommended for Muscat comprises:

- Trunk services: bus routes which provide public transport services along the main mobility corridors of Muscat. Trunk lines are the base for the future development and growth of the system and are the spinal for the provision of public transport.

- Feeder services will operate on medium-density areas, serving the end-of-route terminals and intermediate stations of the trunk services. These services will be established with a zig-zag pattern on minor roads, with shorter stop distances, lower commercial speed and smaller sized vehicles.

- Direct services: in the case that a high demand is detected between two specific areas of Muscat, direct services may be established.

The main advantages of this kind of network:

- Less number of routes is needed to link the same number of destinations.

- Easy to understand by users and easy to use.

- Easy to adapt to new growths.

- Great number of travel patterns.

- This type of network will facilitate the future introduction mass rapid transit systems (BRT or LRT), since users have already adapted their travel patterns.

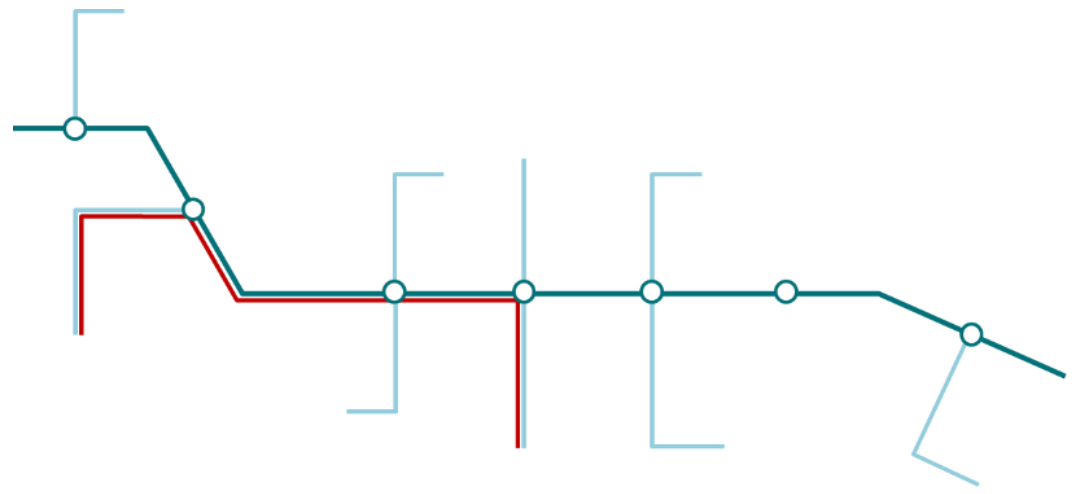

Figure 5 - Proposed structure for the urban bus network

\subsection{Reorganization of the taxi and microbus sector}

Complementing public transport, the PTMP recommended the restructure of the taxi sector, in order to achieve higher quality services with more reliability: 
- Creating an Executive taxi association that provides higher quality services in the most relevant areas such as in the Muscat International Airport, hotels, Sultan Qaboos Port, etc. Also, this association would manage the telephone and internet booking of taxis.

- Implementing new regulation with new licenses (to the vehicle, to the driver), stronger requirements in terms of quality and level of service, further control of taxi drivers, etc.

This reorganization will allow the professionalization of the taxi services and the migration of existing users, part of them to the professionalised taxi services and the other part to the implemented public transport services.

\subsection{Additional proposals to support the success of the system}

Finally, the Plan comprises a number of additional proposals aimed at enhancing public transport, deemed necessary to guarantee its success. A great number of additional proposals are mentioned in the PTMP, the most relevant ones are the following:

- Densification of urban areas and land use integration with public transport.

- Improvement in pedestrian spaces to promote walking and facilitate the access and egress to public transport.

- Car use discourage by parking spaces management and increasing vehicle occupancy.

These additional proposals are not strictly under the responsibility of the MOTC, but also to a number of other organisations, such the Muscat Municipality, Ministry of Housing, Supreme Council for Planning, etc.

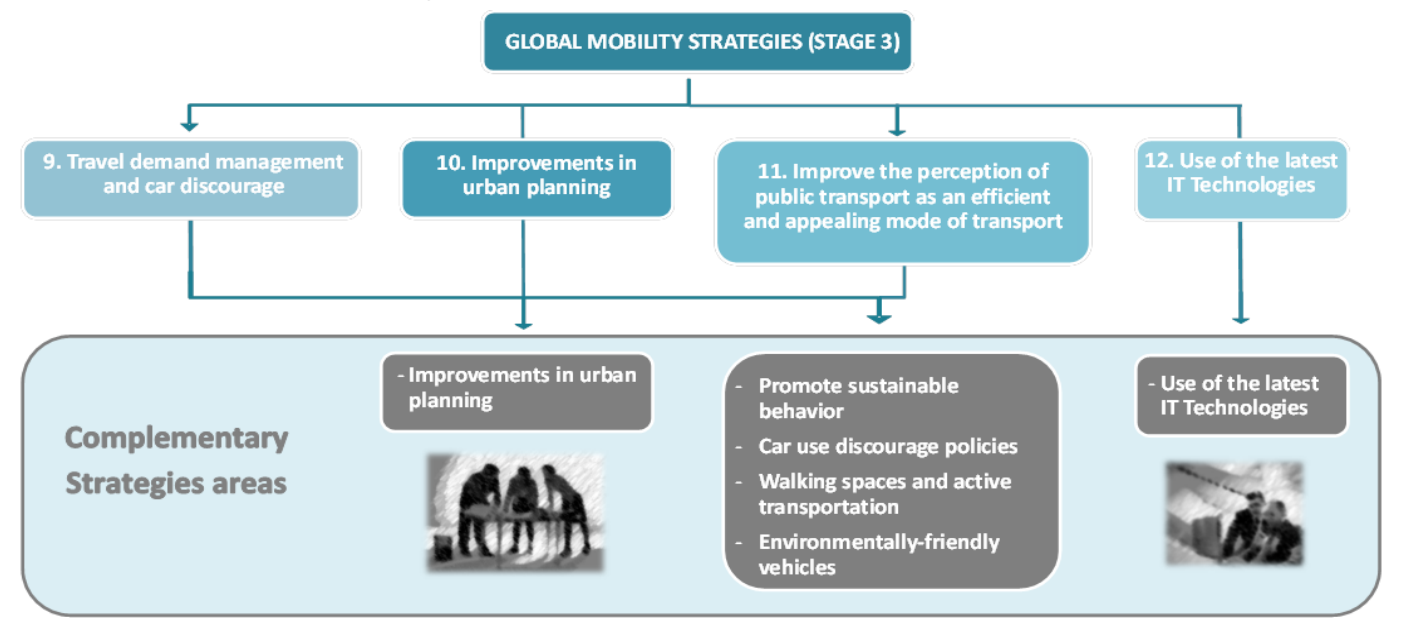

Figure 6 - Complementary strategies areas and their global mobility strategies

\section{ACKNOLEDGEMENTS}

The Directorate General of Planning and Studies of the Ministry of Transport and Communications of Oman; Steering Committee created to follow-up the project: Supreme Council for Planning of Oman, Muscat Municipality, Oman National Transport Company, Oman National Ferries Company, Ministry of Tourism of Oman and, of course, several departments within the Ministry of Transport and Communications of Oman. 